9. Ghinai I, Woods S, Ritger KA, McPherson TD, Black SR, Sparrow L, et al. Community Transmission of SARS-CoV-2 at Two Family Gatherings - Chicago, Illinois, FebruaryMarch 2020. MMWR Morb Mortal Wkly Rep. 2020;69:446-50. https:/ / doi.org/10.15585/mmwr.mm6915e1

10. Somsen GA, van Rijn C, Kooij S, Bem RA, Bonn D. Small droplet aerosols in poorly ventilated spaces and SARS-CoV-2 transmission. Lancet Respir Med. 2020;8:658-9. https://doi.org/10.1016/S2213-2600(20)30245-9

Address for correspondence: Le Van Tan, Oxford University Clinical Research Unit, Vo Van Kiet, District 5, Ho Chi Minh City, Vietnam; email: tanlv@oucru.org

\section{Racial and Workplace Disparities in Seroprevalence of SARS-CoV-2, Baton Rouge, Louisiana, USA}

\author{
Amy K. Feehan, Cruz Velasco, Daniel Fort, \\ Jeffrey H. Burton, Eboni G. Price-Haywood, \\ Peter T. Katzmarzyk, Julia Garcia-Diaz, \\ Leonardo Seoane
}

\begin{abstract}
Author affiliations: Ochsner Clinic Foundation, New Orleans, Louisiana, USA (A.K. Feehan, C. Velasco, D. Fort, J.H. Burton, E.G. Price-Haywood, J. Garcia-Diaz, L. Seoane); The University of Queensland Faculty of Medicine, Ochsner Clinical School, New Orleans (A.K. Feehan, E.G. Price-Haywood, J. Garcia-Diaz, L. Seoane); Pennington Louisiana State University, Baton Rouge, Louisiana, USA (P.T. Katzmarzyk); Louisiana State University Health Sciences Center-Shreveport, Shreveport, Louisiana, USA (L. Seoane)
\end{abstract}

\section{DOI: https://doi.org/10.3201/eid2701.203808}

By using paired molecular and antibody testing for severe acute respiratory syndrome coronavirus 2 infection, we determined point prevalence and seroprevalence in Louisiana, USA, during the second phase of reopening. Infections were highly variable by race and ethnicity, work environment, and ZIP code. Census-weighted seroprevalence was $3.6 \%$, and point prevalence was $3.0 \%$.

W e previously reported results from a seroprevalence study conducted in New Orleans, Louisiana, USA, which was hit hard early in the coronavirus disease (COVID-19) pandemic (1). Baton Rouge is a large metropolitan area roughly 80 miles northwest of New Orleans; at the time of this study, it was in the second phase of reopening after a stay-at-home order. Although the seroprevalence in New Orleans (6.9\%) (1) was similar to prevalence recorded in Spain (5\%), São Paulo, Brazil (4.7\%), and New York, USA (6.9\%) (2,3; B.H. Tess, unpub. data, https://doi.org/10.1101/20 20.06.29.20142331), Baton Rouge had only 3,427 more cases as of August 2, 2020 (17,093 cases), than New Orleans did by May 16, 2020 (13,666 cases) (4). This latest study estimated severe acute respiratory syndrome coronavirus 2 (SARS-CoV-2) infections in the greater Baton Rouge area (Ascension, East Baton Rouge, Livingston, and West Baton Rouge Parishes), with additional information on potential workplace exposures.

The protocol was approved by the Ochsner institutional review board and was designed to enroll and test $\leq 2,500$ participants at 13 sites throughout Baton Rouge during July 15-31. Recruitment targeted a representative sample by using a method developed by Public Democracy (https://www.publicdemocracy. io) and described elsewhere $(1,5)$. In contrast to the New Orleans study, in which persons tested were under a stay-at-home order, Baton Rouge was in phase 2 of reopening. A randomized subset of 500,000 Baton Rouge residents were targeted with digital ads for recruitment. Of those, 3,687 volunteers were recruited and restratified according to census designations; 2,309 were invited to participate, 2,179 enrolled and completed testing, and 2,138 were included in our final analysis. A total of 38 persons were excluded because they lived in ineligible ZIP codes, and 3 withdrew consent (Appendix Figure 1, https://wwwnc.cdc.gov/ EID/article/27/1/20-3808-App1.pdf). All study materials were provided in English, Spanish, and Vietnamese. Participants were offered free transportation. Research staff verbally obtained consent from participants and electronically documented consent and survey responses. We then procured blood samples and nasopharyngeal swab specimens from participants.

We used US Food and Drug Administration Emergency Use Authorization-approved tests. Realtime reverse transcription PCR of nasopharyngeal swab specimens was performed by using the Abbott m2000 RealTime system (Abbott, https:/ / www.molecular.abbott). Qualitative IgG blood tests were performed by using the ARCHITECT i2000SR (Abbott). The IgG test meets criteria established by the Centers for Disease Control and Prevention to yield high positive predictive value, which was validated by Ochsner Health laboratory and others $(6,7)$. Study participants who tested positive on either or both tests were 
Table. Prevalence of past and present severe acute respiratory syndrome coronavirus 2 infections by race and ethnicity across Baton Rouge, Louisiana, after phased reopening, July $2020^{*}$

\begin{tabular}{|c|c|c|c|c|c|c|}
\hline Race or ethnicity & $\begin{array}{l}\text { Positive no./total } \\
\text { no. (\% of sample) }\end{array}$ & $\begin{array}{l}\text { Residents }>18 \\
y, \text { no. ( } \% \text { of } \\
\text { population) }\end{array}$ & $\begin{array}{l}\text { Any infection, } \\
\text { raw, } \dagger \%(95 \% \\
\mathrm{Cl})\end{array}$ & $\begin{array}{l}\text { Any infection, } \\
\text { weighted, } \neq \% \\
(95 \% \mathrm{Cl})\end{array}$ & $\begin{array}{c}\text { Weighted point } \\
\text { prevalence,§ \% } \\
(95 \% \mathrm{Cl})\end{array}$ & $\begin{array}{c}\text { Weighted } \\
\text { seroprevalence, } \\
\%(95 \% \mathrm{Cl}) \\
\end{array}$ \\
\hline Total & & $551,185(100)$ & $6.0(5.0-7.1)$ & $6.6(5.7-7.7)$ & $3.0(2.3-3.7)$ & $3.6(2.8-4.4)$ \\
\hline & & 2 & & & & \\
\hline can & & 7 & 1.0 & 11. & 3.5 & 7.5 \\
\hline & & & & & & 1.7 \\
\hline & & 7,0 & 3.6 & & & 2.7 \\
\hline $\begin{array}{l}\text { Hispanic or Latino, } \\
\text { any race }\end{array}$ & & 20,125 & $3.5(7.6-$ & 11.8 & ) & $-4.0)$ \\
\hline \multicolumn{7}{|c|}{$\begin{array}{l}\text { *Census } 2018 \text { population estimates. By August 2, 2020, a total of 17,093 state-aggregated, confirmed cases had been reported in Ascension, East Baton } \\
\text { Rouge, Livingston, and West Baton Rouge parishes (4). } \\
\text { †Percentage of sample with a PCR-positive test, an IgG-positive test, or both. } \\
\text { †Census-weighted percentage of PCR-positive test, IgG-positive test, or both calculated to match } 2018 \text { racial demographics by parish and combined. } \\
\text { §Census-weighted percentage of PCR-positive and IgG-negative tests calculated to match } 2018 \text { racial demographics by parish and combined. } \\
\text { TCensus-weighted percentage of IgG-positive tests calculated to match } 2018 \text { racial demographics by parish and combined. } \\
\text { \#Other includes American Indian or Alaska Native, Pacific Islander, and multiracial persons. }\end{array}$} \\
\hline
\end{tabular}

assessed as having been infected with SARS-CoV-2. Point estimates and corresponding 95\% CIs for proportions of SARS-CoV-2 exposure (PCR+ or IgG+ tests), point prevalence (PCR+, IgG-), and seroprevalence (IgG+ tests regardless of PCR test result) were estimated for the Baton Rouge area by using raw and census-weighted counts. Unadjusted odds ratios with Firth correction were calculated for all variables.

The sample was $63.6 \%$ female and $66.9 \%$ white; average age was 48.7 years (range 18-91) and average household size 2.84 persons. The census-weighted estimate of SARS-CoV-2 infections in the sample is $6.6 \%$ $(6.0 \%$, raw), with $3.0 \%$ positive for active viral shedding without detectable antibody, which translates to 16,536 contagious persons. By race and ethnicity, seroprevalence was highest (7.5\%) in Black participants, compared with White non-Hispanic (1.8\%), Asian non-Hispanic $(1.7 \%)$, Hispanic of any race $(1.6 \%)$, and other $(2.7 \%)$ participants (Table).

The point prevalence and any SARS-CoV-2 infection were mapped by ZIP codes across the greater Baton Rouge area (Appendix Figure 2). Point prevalence and all infections were highly variable by ZIP code.

Marital status was associated with prevalence $\left(p=0.0005\right.$ by $x^{2}$ test). Single persons had the highest rate of infection $(9.3 \%)$, compared with rates for married or cohabitating participants (5.0\%), and were 1.9 times more likely to test positive (Figure). Work environment also affected prevalence $\left(p=0.01\right.$ by $\chi^{2}$ test); the lowest prevalence was in participants who worked from home part-time and went to a workplace part-time $(3.7 \%)$. Those who worked primarily outside the home had the highest prevalence $(8.2 \%)$ and were 2.3 times more likely to test positive than those who worked from home at least part-time. Infection rates varied by occupation ( $p=0.01$ by $x^{2}$ test); the lowest positivity was in office workers $(3.0 \%)$ and in- creased odds of testing positive occurred in delivery, healthcare, and other public-facing jobs. However, based on seroprevalence, which also varied substantially by occupation ( $p=0.03$ by $\chi^{2}$ test), healthcare workers and public-facing workers bore the brunt of early infections, as demonstrated by higher odds of testing positive for antibodies (Figure).

We found the prevalence of SARS-CoV-2 infection in Baton Rouge to be $6.6 \%$ but with a heavy concentration of new, contagious infections (3.0\%). Persons who were infected early possibly no longer had antibodies. This finding differed from our New Orleans study, which was performed after extensive lockdowns and estimated new infections at $0.9 \%$ (1). Some populations had higher rates of infection than others, including Black and Hispanic communities and public-facing workers or those who do not work from home.

\section{Acknowledgments}

The authors would like to especially thank the laboratories at the Ochsner Medical Center Jefferson Highway Campus for testing and keeping track of research samples; Christy Reeves for liaising with public leaders and sites; Eric Sapp and Dan Nichols for their recruitment effort; Susan Green, Charlene Ho, Lena Hooper, Patty Kline, and Candace Melancon for clinical site management; Johanna Veal, Lyndsey Buckner-Baiamonte, Ansley Hammons, and Ashley LaRoche for research site management; and countless research coordinators, clinical staff, marketing personnel, medical students, and Epic and IT staff for making site testing possible. The Ochsner Health Market Planning and Analysis team designed the maps in Appendix Figure 1. The authors thank Kathleen McFadden for her thorough editing. We would like to thank the Ochsner Language Services Department for helping to increase inclusivity and to acknowledge the East Baton 

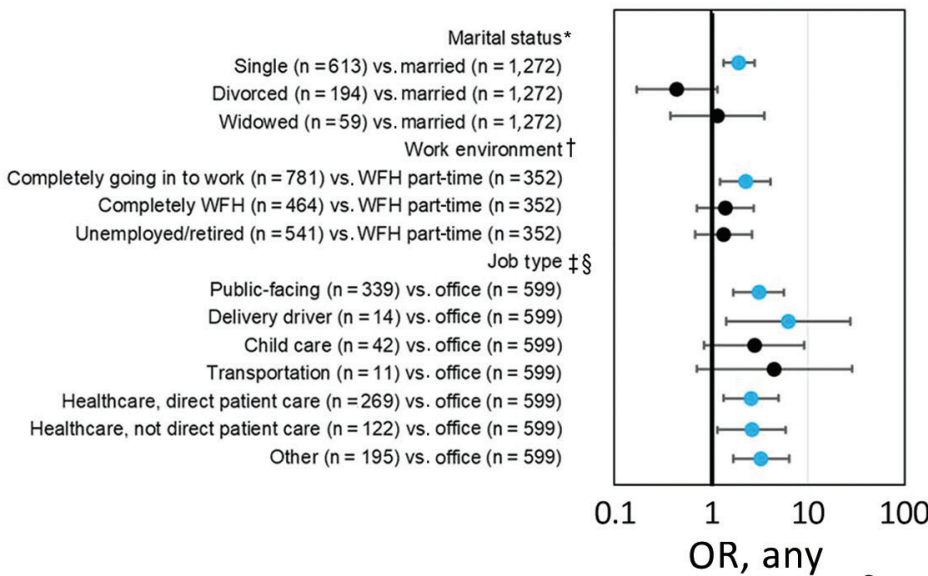

positive test§

Women $(n=1,361)$ vs. men $(n=777)$ Race/ethnicity**

Black NH $(n=516)$ vs. White NH $(n=1,431)$ Asian $\mathrm{NH}(n=60)$ vs. White $\mathrm{NH}(n=1,431)$

$\geq 2 \mathrm{NH}(\mathrm{n}=20)$ vs. White $\mathrm{NH}(\mathrm{n}=1,431)$

Hispanic (any race) $(n=104)$ vs. $\mathrm{NH}(n=2,034)$

Other $\mathrm{NH}(n=7)$ vs. White $\mathrm{NH}(n=1,431)$

$18-19(n=35)$ vs. $\geq 70(n=179)$

$20-29(n=222)$ vs. $\geq 70(n=179)$

$30-39(n=428)$ vs. $\geq 70 \quad(n=179)$

$40-49(n=434)$ vs. $\geq 70(n=179)$

$50-59(n=461)$ vs. $\geq 70(n=179)$

$60-69(n=379)$ vs. $\geq 70(n=179)$

Do you think you've had COVID-19? $(n=520)$

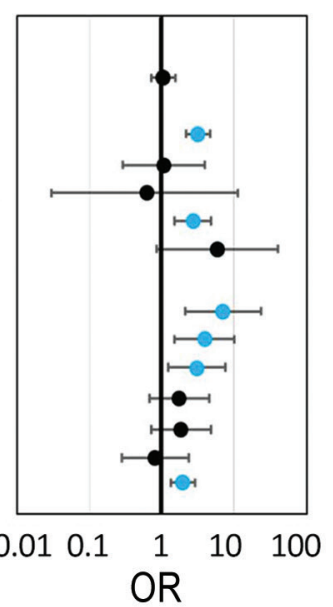

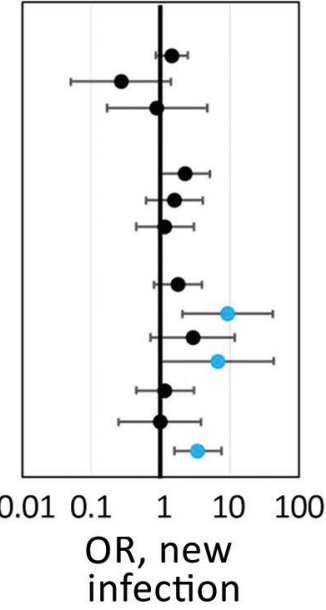
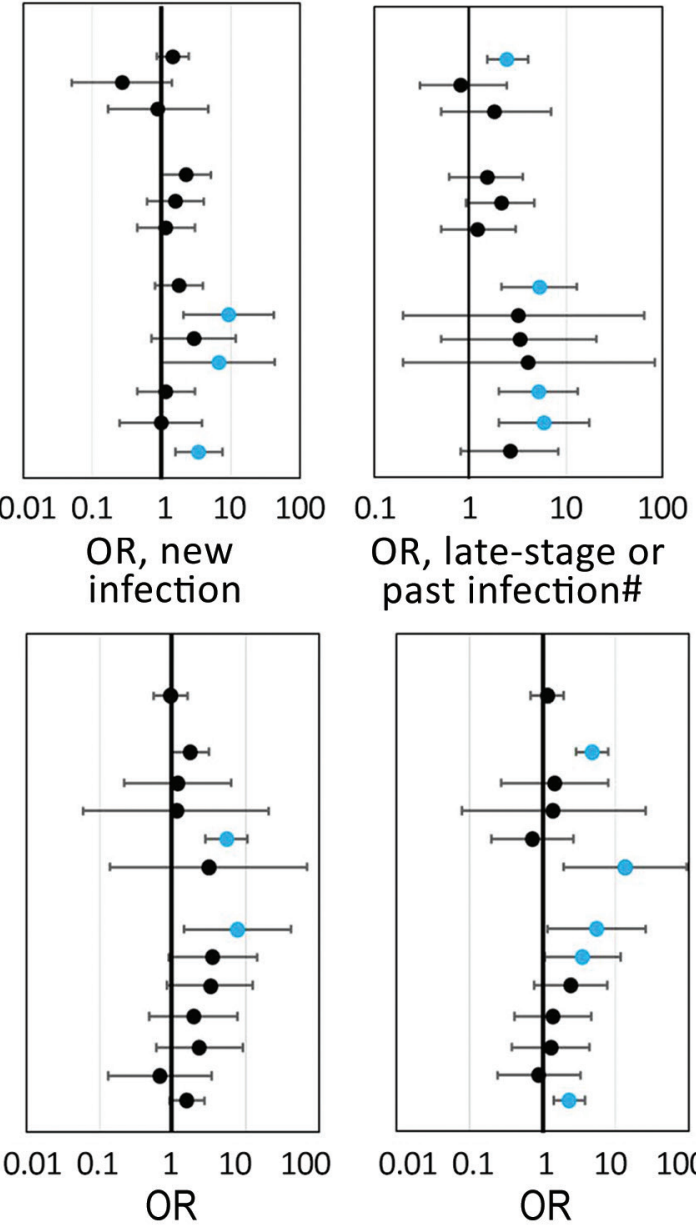

Figure. Odds ratios of severe acute respiratory syndrome coronavirus 2 infections by marital status, work environment, and job type after phased reopening in Baton Rouge, Louisiana, USA, July 2020. OR from unweighted logistic regression without covariates with Firth correction are shown with $95 \%$ Cls. Reference categories' percent positivity are married (5.0\% any infection, $2.3 \%$ seroprevalence), WFH part-time (3.7\% any infection, $2.0 \%$ seroprevalence), and office workers (3.0\% any infection, $1.0 \%$ seroprevalence). WFH, work from home; $\mathrm{NH}$, non-Hispanic; OR, odds ratio. *Odds of any infection $(p=0.0005)$ and seroprevalence $(p=0.03)$ differ by marital status. †Odds of any infection $(p=0.01)$ differ by work environment. $\ddagger$ Odds of any infection $(p=0.01)$ and seroprevalence $(p=0.03)$ differ by job type. $\$$ Six people did not give an answer for job type; none tested positive on any test. Unemployed/retired people $(n=541)$ are not included in this category. ๆPercentage and OR of any positive test (PCR+ or lgG+). \#Percentage and OR of late-stage or past infections (lgG+, regardless of PCR status). ${ }^{* *}$ Odds of any infection $(p<0.0001)$ and seroprevalence $(p<0.0001)$ differ by race and ethnicity. $\dagger+O d d s$ of any infection $(p<0.0001)$ and seroprevalence $(p=0.0074)$ differ by age.

Rouge Mayor-President Sharon Weston Broome and Pennington Biomedical Research Center for their collaboration and support of this project.

The study was funded by the Baton Rouge Area Foundation, Louisiana COVID-19 Health Equity Task Force, and the Humana Foundation, with additional support from the Blue Cross and Blue Shield of Louisiana Foundation, Healthy Blue, the Huey and Angelina Wilson Foundation, and the Irene W. and C.B. Pennington Foundation. The funders had no role in study design, data collection and analysis, decision to publish, or preparation of the manuscript.

\section{About the Author}

Dr. Feehan is a research scientist at the Ochsner Clinic Foundation's Infectious Disease Clinical Research Department. Her research focuses on the gut microbiome as a treatment modality for neurologic disease, but more immediately on the COVID-19 pandemic.

\section{References}

1. Feehan AK, Fort D, Garcia-Diaz J, Price-Haywood EG, Velasco C, Sapp E, et al. Seroprevalence of SARS-CoV-2 and infection fatality ratio, Orleans and Jefferson parishes, Louisiana, USA, May 2020. Emerg Infect Dis. 2020;26:2766-9. https:// doi.org/10.3201/eid2611.203029 
2. Pollán M, Pérez-Gómez B, Pastor-Barriuso R, Oteo J, Hernán MA, Pérez-Olmeda M, et al.; ENE-COVID Study Group. Prevalence of SARS-CoV-2 in Spain (ENE-COVID): a nationwide, population-based seroepidemiological study. Lancet. 2020;396:535-44. https:/ / doi.org/10.1016/ S0140-6736(20)31483-5

3. Havers FP, Reed C, Lim T, et al. Seroprevalence of Antibodies to SARS-CoV-2 in 10 sites in the United States, March 23-May 12, 2020. JAMA Intern Med. 2020 Jul 21 [Epub ahead of print].

4. Louisiana Department of Public Health. Louisiana coronavirus COVID-19 information [cited 2020 Sep 20]. http://ldh.la.gov/Coronavirus

5. Feehan A, Fort D, Garcia-Diaz J, Price-Haywood E, Velasco C, Sapp E, et al. Frequency of symptoms and asymptomatic SARS-CoV-2 infection in New Orleans, Louisiana after 7 weeks of a stay-at-home order [cited 2020 Sep 8]. https:/ / ssrn.com/abstract=3633166 https:/ / doi.org/ $10.2139 /$ ssrn.3633166

6. US Centers for Disease Control and Prevention. Interim guidelines for COVID-19 antibody testing [cited 2020 Aug 10]. https:/ / www.cdc.gov/coronavirus/2019-ncov/lab/ resources/antibody-tests-guidelines.html

7. Bryan A, Pepper G, Wener MH, Fink SL, Morishima C, Chaudhary A, et al. Performance characteristics of the Abbott Architect SARS-CoV-2 IgG assay and seroprevalence in Boise, Idaho. J Clin Microbiol. 2020;58:e00941-20. https://doi.org/10.1128/JCM.00941-20

Address for correspondence: Amy Feehan, Clinical Infectious Diseases Research, $1^{\text {st }}$ Fl AT, 1514 Jefferson Hwy, Jefferson, LA 70121, USA; email: amy.feehan@ochsner.org

\section{SARS-CoV-2 Cluster in Nursery, Poland}

\author{
Magdalena Okarska-Napierała, Joanna Mańdziuk, \\ Ernest Kuchar
}

Author affiliation: Medical University of Warsaw, Warsaw, Poland

DOI: https://doi.org/10.3201/eid2701.203849

We report a cluster of surprisingly high spread of severe acute respiratory syndrome coronavirus 2 (SARS-CoV-2) associated with a single nursery in Poland. Our findings contrast with the presumed negligible role of children in driving the SARS-CoV-2 pandemic. Children 1-2 years of age might be effective SARS-CoV-2 spreaders.
Despite robust research, knowledge about coronavirus disease (COVID-19) spread and effective control measures is still limited. Until recently, research has indicated that children rarely spread the infection to adults and are not the primary drivers of severe acute respiratory syndrome coronavirus 2 (SARS-CoV-2) transmission (1).

We describe characteristics of the cluster of SARSCoV-2 cases that emerged in a single nursery in Poland within 2 weeks of its reopening. We anonymized all data and collected no sensitive data. The Bioethics Committee of the Medical University of Warsaw approved the study protocol.

The nursery at issue was reopened after a nationwide lockdown on May 18, 2020. On May 31, a nursery worker reported family contact with a symptomatic SARS-CoV-2-infected person, and the nursery was closed. During the 14 days the nursery was open, a mean of 25 children attended the nursery daily. Children spent $\approx 8$ hours there, divided into 3 groups, each cared for by 2 caregivers (Appendix, https:// wwwnc.cdc.gov/EID/article/27/1/20-3849-App1. pdf). Neither children nor caregivers moved across multiple classes. Caregivers wore facemasks when in contact with children. Parents did not enter the building when dropping off and picking up children. Contacts between parents and nursery workers lasted $<15$ minutes, with facemasks on. Family members of different children did not mix.

The index case of SARS-CoV-2 infection (in a nursery worker with family contact) was confirmed on June 4. Subsequent PCR testing of nursery staff, children attending the facility, and family members (2 initial case-patients plus 104 other persons) (Appendix) revealed positive results in an additional 4 nursery workers (of whom 1 was also a parent of a child attending the facility), 3 children of the nursery workers, 8 children attending the facility, 3 siblings of those children, 8 parents, and 1 grandparent. The cluster involved a total of 29 persons; 8 were children attending the nursery, and 12 were children's family members who did not enter the facility (Table). One child with a negative result had 2 parents with positive results. One child's parent tested negative in this cluster but had tested positive within the previous 2 weeks, involved in another cluster.

The overall positivity rate in our cluster was $27 \%$. COVID-19 prevalence in Poland is low. The number of tests conducted in the country was 124,194 in June, whereas the number of positive cases was 1,374, which corresponded to a positivity rate of $1 \%$ (2). Thus, local SARS-CoV-2 circulation in society is not sufficient to explain the positivity rate in our cluster. 